\title{
Competition of coarsening and shredding of clusters in a driven diffusive lattice gas
}

\author{
Ambarish Kunwar ${ }^{1} \ddagger$, Debashish Chowdhury ${ }^{2} \S$, Andreas \\ Schadschneider ${ }^{3} \|$ and Katsuhiro Nishinari ${ }^{4}$ व \\ ${ }^{1}$ Department of Physics, Indian Institute of Technology, Kanpur 208016, India. \\ ${ }^{2}$ Department of Physics, Indian Institute of Technology, Kanpur 208016, India. \\ ${ }^{3}$ Institute for Theoretical Physics, University of Cologne, D-50937 Köln, Germany. \\ ${ }^{4}$ Department of Aeronautics and Astronautics, Faculty of Engineering, University of \\ Tokyo, Hongo, Bunkyo-ku, Tokyo 113-8656, Japan.
}

\begin{abstract}
We investigate a driven diffusive lattice gas model with two oppositely moving species of particles. The model is motivated by bi-directional traffic of ants on a pre-existing trail. A third species, corresponding to pheromones used by the ants for communication, is not conserved and mediates interactions between the particles. Here we study the spatio-temporal organization of the particles. In the uni-directional variant of this model it is known to be determined by the formation and coarsening of "loose clusters". For our bi-directional model, we show that the interaction of oppositely moving clusters is essential. In the late stages of evolution the cluster size oscillates because of a competition between their 'shredding' during encounters with oppositely moving counterparts and subsequent "coarsening" during collisionfree evolution. We also establish a nontrivial dependence of the spatio-temporal organization on the system size.

PACS numbers: 45.70.Vn, 02.50.Ey, 05.40.-a
\end{abstract}

\section{Introduction}

Systems of interacting driven particles are of current interest in statistical physics for understanding the fundamental principles of spatio-temporal organization far from equilibrium as well as for possible wide ranging practical applications [1, 2]. The simplest model of this type with only one species of particles is the so-called totally asymmetric simple exclusion process (TASEP). In the TASEP, a particle is picked up randomly and moved forward by one lattice spacing, with the hopping probability $q_{+}$, provided the target site on the one-dimensional lattice is empty.

Most of the earlier models with more than one species of particles [3, 4, 5, 6, 7, 8, 9, 10] addressed mainly the questions on the (im)possibility of spontaneous symmetry

‡ E-mail: ambarish@iitk.ac.in

$\S$ E-mail: debch@iitk.ac.in

|| E-mail: as@thp.uni-koeln.de

ฯ E-mail: tknishi@mail.ecc.u-tokyo.ac.jp 


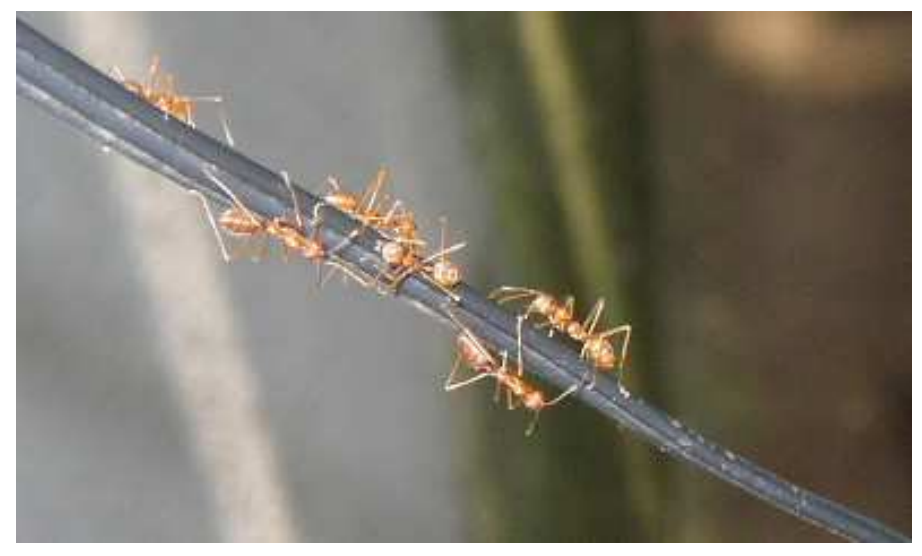

Figure 1. A snapshot of an ant-trail on a hanging cable. It can be regarded as strictly one-dimensional. But, nevertheless, traffic flow in opposite directions is possible as two ants, which face each other on the upper side of the cable, can exchange their positions if one of them, at least temporarily, switches over to the lower side of the cable.

breaking, phase separation, etc. in one-dimensional stochastic driven systems. Here we extend the two-species models like, for example, ABC model [4, 5] and AHR model [6] to develop a three-species model [1]. The density of the new species introduced in our model, which corresponds to the pheromones secreted by the ants for communication, is not conserved by the dynamics of the system and its coupling to the two other conserved variables leads to uncommon features of the spatio-temporal organisations of the conserved particles. For a special choice of the model parameters our model becomes identical to the AHR model classes (see Sec. 3).

Our model is motivated by a real-life situation where bi-directional ant-traffic in an effectively one-dimensional system is observed. Fig. 1 shows a hanging cable which is part of an ant-trail. Ants prefer moving on the upper side of the cable, which would make the motion strictly one-dimensional. If ants moving in opposite directions meet head-on, after short "negotiations" one of them would switch to the lower part of the cable temporarily in order to make passing possible. Due to the "negotiations" this process leads to slowing down of both the ants. We capture this entire process by an exchange of the positions of the two ants with a sufficiently low probability which corresponds to a slower movement of each of them as compared to a freely moving ant. The mathematical formulation of the model in terms of such exchange probabilities is very similar to the formulations of the class of one-dimensional stochastic models of driven-diffusive lattice gases to which the AHR and ABC models belong.

The number of particles leaving a site per unit time is called the flux or current; the flux-versus-density relation is often referred to as the fundamental diagram. We study interplay of spatio-temporal organization and flow (fundamental diagram) in the system by a combination of analytical arguments and extensive computer simulations. 


\begin{tabular}{|c|c|c|}
\hline initial & final & rate \\
\hline $\mathrm{RL}$ & $\mathrm{RL}$ & $1-K$ \\
& $\mathrm{LR}$ & $K$ \\
\hline $\mathrm{RP}$ & $\mathrm{RP}$ & $(1-f)(1-Q)$ \\
& $\mathrm{R} 0$ & $f(1-Q)$ \\
& $0 \mathrm{R}$ & $f Q$ \\
& $\mathrm{PR}$ & $(1-f) Q$ \\
\hline $\mathrm{R} 0$ & $\mathrm{R} 0$ & $1-q$ \\
& $0 \mathrm{R}$ & $f q$ \\
& $\mathrm{PR}$ & $(1-f) q$ \\
\hline $\mathrm{PR}$ & $\mathrm{PR}$ & $1-f$ \\
& $0 \mathrm{R}$ & $f$ \\
\hline $\mathrm{P} 0$ & $\mathrm{P} 0$ & $1-f$ \\
& 00 & $f$ \\
\hline $\mathrm{PP}$ & $\mathrm{PP}$ & $(1-f)^{2}$ \\
& $\mathrm{P} 0$ & $f(1-f)$ \\
& $0 \mathrm{P}$ & $f(1-f)$ \\
& 00 & $f^{2}$ \\
\hline
\end{tabular}

Figure 2. Nontrivial transitions and their transition rates. Transitions from initial states $P L, 0 L$ and $0 P$ are not listed. They can be obtained from those for $L P, L 0$ and $P 0$, respectively, by replacing $R \leftrightarrow L$ and, then, taking the mirror image.

\section{The model}

In our model the right-moving (left-moving) particles, represented by $R(L)$, are never allowed to move towards left (right); these two groups of particles are the analogs of the outbound and nest-bound ants in a bi-directional traffic on the same trail. Thus, no U-turn is allowed. In addition to the TASEP-like hopping of the particles onto the neighboring vacant sites in the respective directions of motion, the $R$ and $L$ particles on nearest-neighbour sites and facing each other are allowed to exchange their positions, i.e., the transition $R L \rightarrow L R$ takes place, with the probability $K$. This might be considered as a minimal model for the motion of ants on a hanging cable as shown in Fig. 1. When a outbound ant and a nest-bound ant face each other on the upper side of the cable, they slow down and, eventually, pass each other after one of them, at least temporarily, switches over to the lower side of the cable. Similar observations have been made for normal ant-trails where ants pass each other after turning by a small angle to avoid head-on collision [17, 16]. In our model, as commonly observed in most real ant-trails, none of the ants is allowed to overtake another moving in the same direction. Ants drop a chemical (generically called pheromone) on the substrate as they move forward [12]. They can follow the trail by picking up the "smell" of the trail pheromone provided the pheromone evaporates sufficiently slowly with time. We now introduce 
a third species of particles, labelled by the letter $P$, which are intended to capture the essential features of pheromone. The $P$ particles are deposited on the lattice by the $R$ and $L$ particles when the latter hop out of a site; an existing $P$ particle at a site disappears when a $R$ or $L$ particle arrives at the same location. The $P$ particles cannot hop but can evaporate, with a probability $f$ per unit time, independently from the lattice. None of the lattice sites can accommodate more than one particle at a time. From now onwards, we shall refer to this model as the PRL model. The state of the system is updated in a random-sequential manner. Because of the periodic boundary conditions, the densities of the $R$ and the $L$ particles are conserved. In contrast, the density of the $P$ particles is a non-conserved variable. The distinct initial states and the corresponding final states for pairs of nearest-neighbor sites are shown in fig 2 together with the respective transition probabilities.

\section{Results}

Suppose $N_{+}$and $N_{-}=N-N_{+}$are the total numbers of $R$ and $L$ particles, respectively. For a system of length $M$ the corresponding densities are $c_{ \pm}=N_{ \pm} / M$ with the total density $c=c_{+}+c_{-}=N / M$. Of the $N$ particles, a fraction $\phi=N_{+} / N=c_{+} / c$ are of the type $R$ while the remaining fraction $1-\phi$ are $L$ particles. The corresponding fluxes are denoted by $F_{ \pm}$. In both the limits $\phi=1$ and $\phi=0$ this model reduces to our earlier model [18, 19], motivated by uni-directional ant-traffic, which is closely related to the bus-route models [20, 21] and the models of pedestrian dynamics [22, 23]. One unusual feature of this PRL model is that the flux does not vanish in the densepacking limit $c \rightarrow 1$. In fact, in the full-filling limit $c=1$, the exact non-vanishing flux $F_{+}=K c_{+} c_{-}=F_{-}$at $c_{+}+c_{-}=c=1$ arises only from the exchange of the $R$ and $L$ particles, irrespective of the magnitudes of $f, Q$ and $q$.

In the special case $Q=q=q_{H}$ the hopping of the ants become independent of pheromone. This special case of the PRL model is identical to the AHR model [6] with $q_{-}=0=\kappa$. A simple homogeneous mean-field approximation (HMFA) yields the estimates

$$
F_{ \pm} \simeq c_{ \pm}\left[q_{H}(1-c)+K c_{\mp}\right]
$$

irrespective of $f$, for the fluxes $F_{ \pm}$at any arbitrary $c$. On the other hand, the exact expression for the flux of the AHR model, parametrized [7] by a rapidity variable $\xi \in\left[0, \frac{1}{(1-a)^{2}}\right]$, is given by:

$$
\begin{aligned}
J(\xi) & =\frac{2 a^{2} \xi}{\mathcal{N}}, \\
\rho(\xi) & =2 \frac{a(1+a) \xi[(1+a) \sqrt{1+4 a \xi}-(1-a)]}{\mathcal{N} \sqrt{1+4 a \xi}}
\end{aligned}
$$

where

$$
\mathcal{N}=1+a^{2}+2 a(1+a)^{2} \xi-\left(1-a^{2}\right) \sqrt{1+4 a \xi}
$$



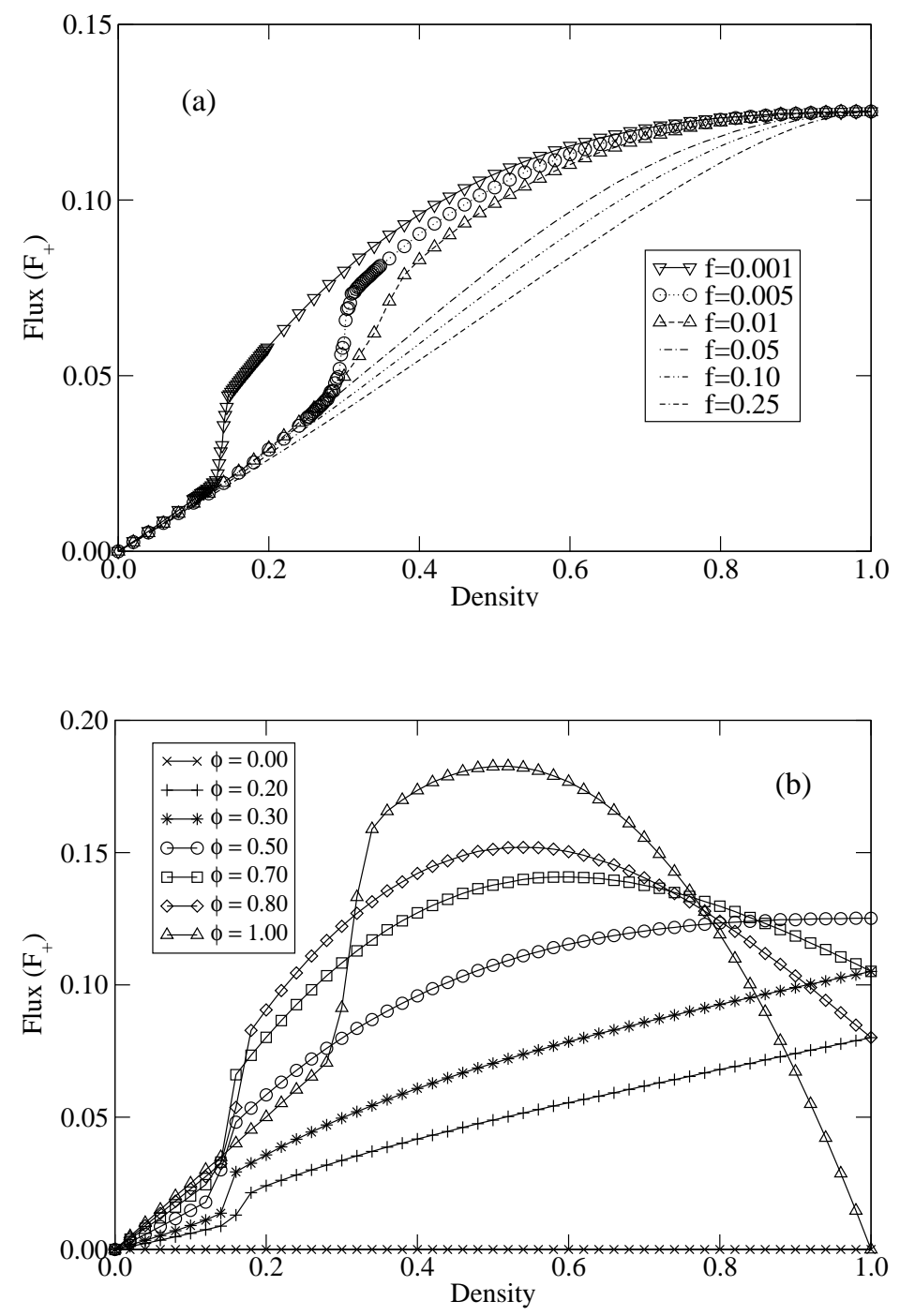

Figure 3. The fundamental diagrams in the steady-state of the PRL model for several different values of (a) $f$ (for $\phi=0.5$ ) and (b) $\phi$ (for $f=0.001$ ). The other common parameters are $Q=0.75, q=0.25, K=0.5$ and $M=1000$.

for $1 / 2 \leq q_{H} \leq 1$, where $a=\frac{1-q_{H}}{q_{H}}$ and the unit of elementary time scale has been set by choosing $K=1$. A comparison of the equation (11) (with $K=1$ ) and the exact result (3) in Fig. (4) shows that the flux in the HMFA, although an underestimate, is a reasonably good approximation for all $q_{H} \geq 1 / 2$. Deviation from the exact values for $q_{H}<1 / 2$ indicates the presence of stronger correlations at smaller values of $q_{H}$. For the generic case $q \neq Q$, the flux in the PRL model depends on the evaporation rate $f$ of the $P$ particles. In Fig. 3 we plot the fundamental diagrams for wide ranges of values of $f$ (in Fig. 3(a)) and $\phi$ (in Fig. 3(b)), corresponding to one set of hopping probabilities. First, note that the data in Figs. 3 are consistent with the physically expected value of $F_{ \pm}(c=1)=K c_{+} c_{-}$, because in the dense packing limit only the exchange of the 


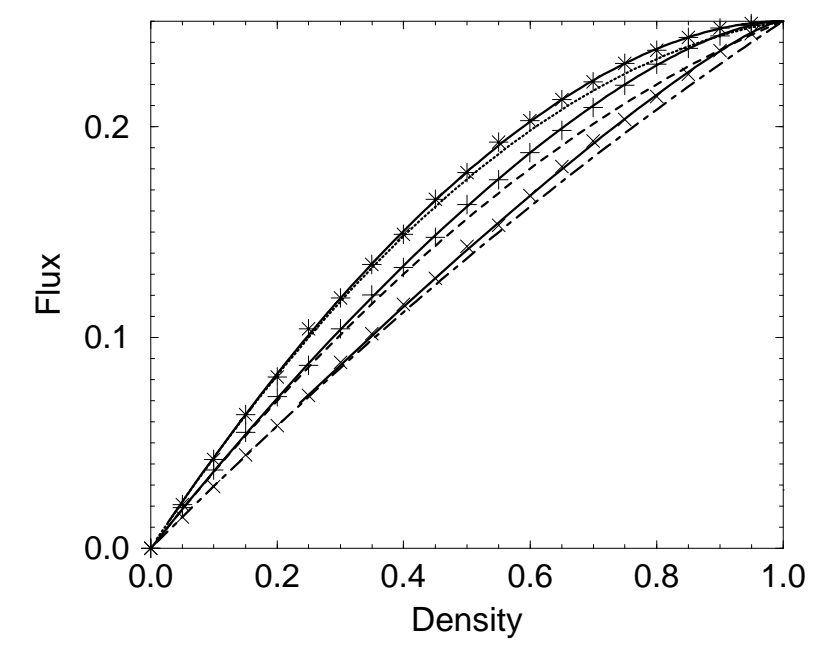

Figure 4. Fundamental diagram in the special case $Q=q=q_{H}$. The continuous curves, marked by $*,+$ and $\times$, are the exact results corresponding to $q_{H}=$ $0.90,0.75,0.60$, respectively. The corresponding HMFA results have been shown by the dotted, dashed and dashed-dotted curves, respectively. The points marked by $*$, + and $\times$ have been obtained from computer simulations.

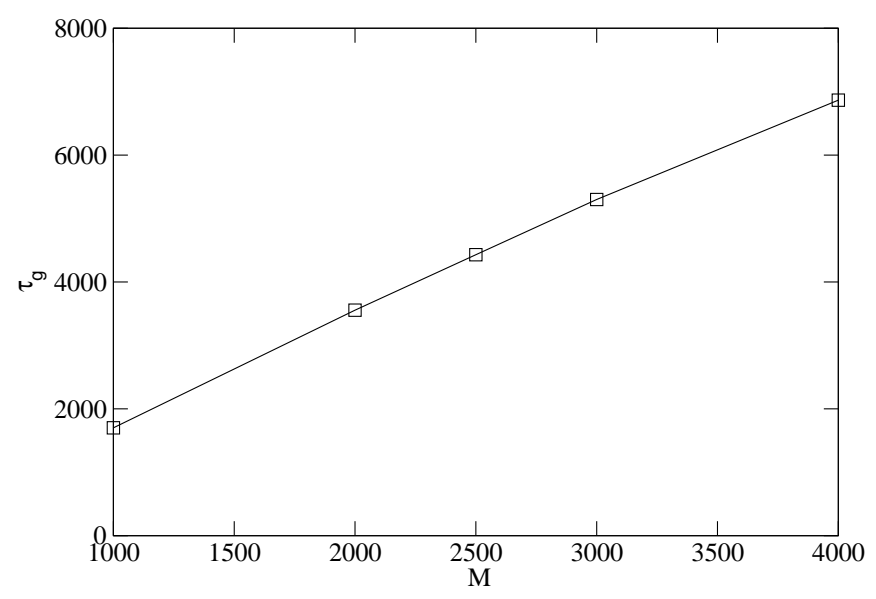

Figure 5. Time gap $\tau_{g}$ between successive collisions as a function of system size $\mathrm{M}$ for $Q=0.75, q=0.25, K=0.5, f=0.005, c=0.2$ and $\phi=0.3$

oppositely moving particles contributes to the flux. Moreover, the sharp rise of the flux over a narrow range of $c$ observed in both Fig. 3 (a) and (b) arise from the nonmonotonic variation of the average speed with density, an effect which was also observed in our earlier model for uni-directional ant traffic [18, 19]. This nonmonotonicity was shown to be a consequence of the formation of so-called loose clusters. These are regions in space where the particle density $c_{\mathrm{lc}}$ is larger than the average density $c$, but not maximal, i.e. $c<c_{\mathrm{lc}}<1$. These loose cluster dominate the behaviour at intermediate particle 


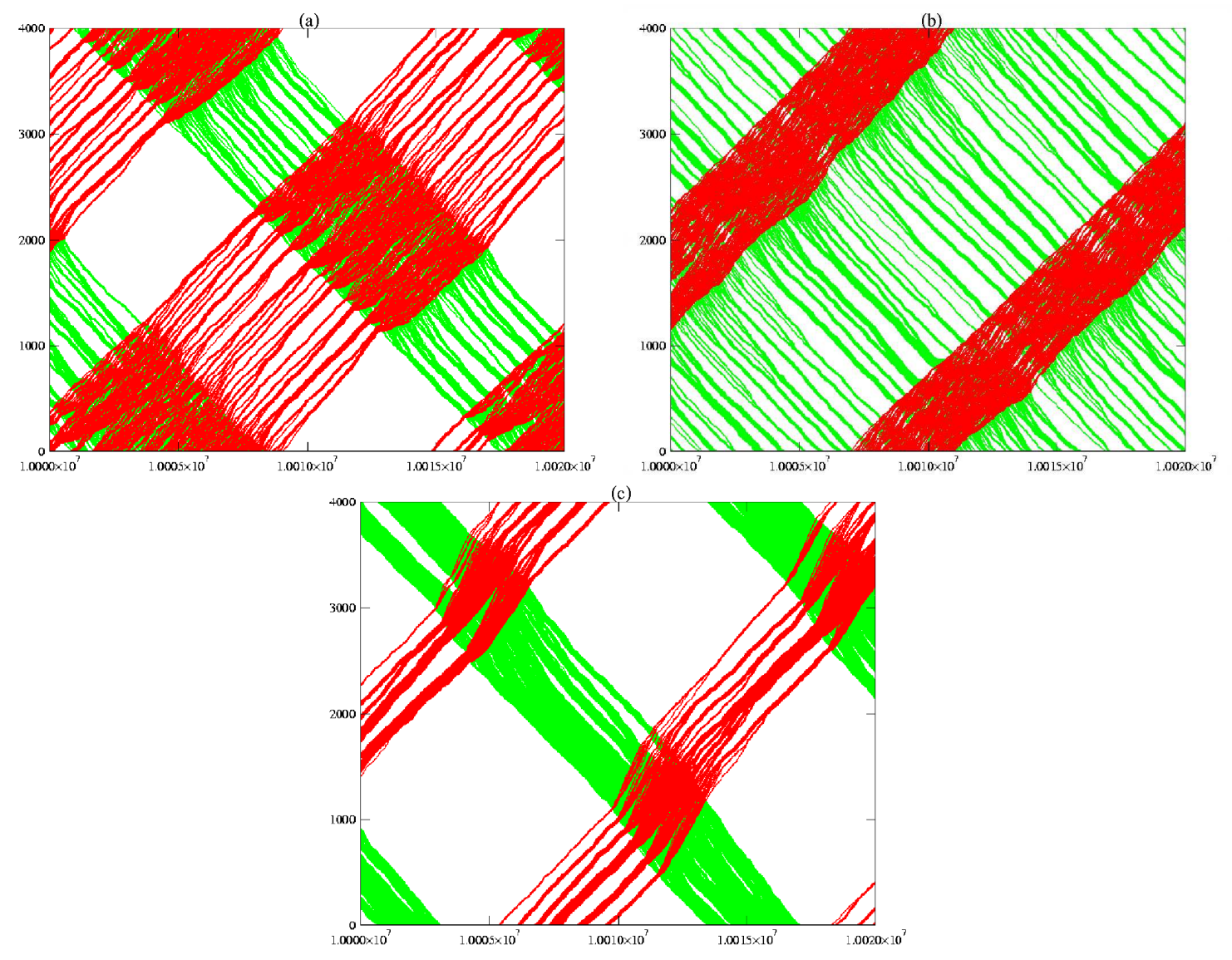

Figure 6. Space-time plot of the PRL model for $Q=0.75, q=0.25, f=0.005$, $M=4000, c=0.2$ and (a) $\phi=0.5, K=0.2$, (b) $\phi=0.3, K=0.2$, (c) $\phi=0.3$, $K=0.5$. The red and green dots represent the right-moving and left-moving ants, respectively.

densities $c$ and small evaporation rates $f$ where they are formed due to effectively longerranged attractive interactions introduced by the pheromones.

In the special limits $\phi=0$ and $\phi=1$, over a certain regime of density (especially at small $f$ ), the particles are known [18, 19] to form "loose" (i.e., non-compact) clusters 19. which are defined to be regions of the system with a density $c_{\ell c}$ that is larger than the average global density, i.e., $c<c_{\ell c}<1$. If the system evolves from a random initial condition at $t=0$, then during coarsening of the cluster, its size $R(t)$ at time $t$ is given by $R(t) \sim t^{1 / 2}[20,21]$. Therefore, in the absence of encounter with oppositely moving particles, $\tau_{ \pm}$, the coarsening time for the right-moving and left-moving particles would grow with system size as $\tau_{+} \sim \phi^{2} M^{2}$ and $\tau_{-} \sim(1-\phi)^{2} M^{2}$.

In the PRL model with periodic boundary conditions, the oppositely moving "loose" clusters "collide" against each other periodically. Let us define $\tau_{g}$ to be the time gap between the successive collisions of the two clusters which is the time interval between the end of one collision and the beginning of the next collision. It is straightforward to see that $\tau_{g}$ increases linearly with the system size following $\tau_{g} \sim M$; we have verified 




(a)

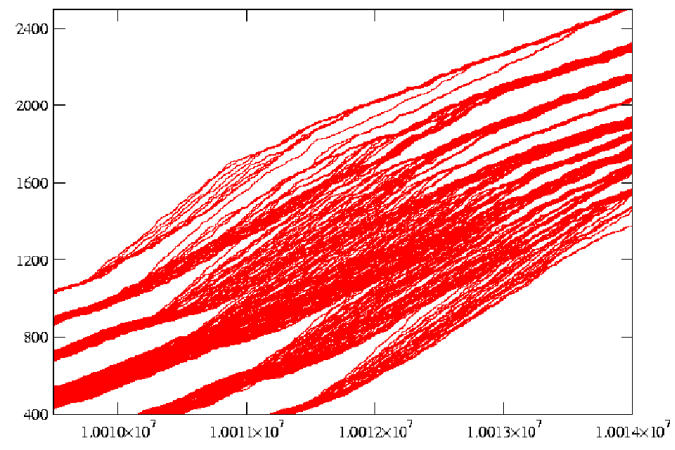

(b)

Figure 7. Magnified view of the first and second collision area respectively of Fig. 6(c)

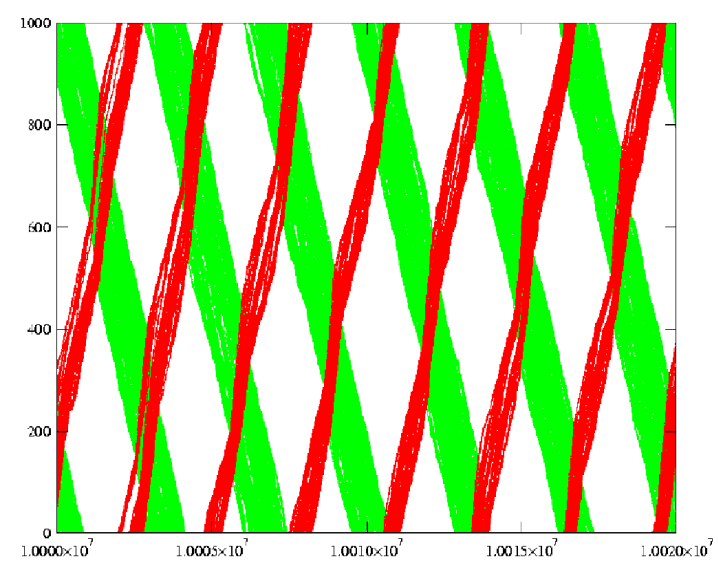

(a)

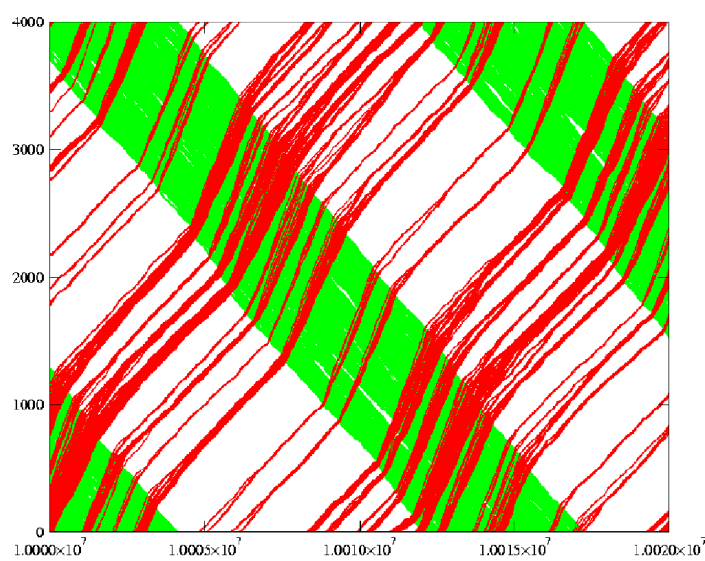

(b)

Figure 8. Space-time plot of the PRL model for $Q=0.50, q=0.25, f=0.005$, $c=0.2, \phi=0.3, K=1.0$ and (a) $M=1000$, (b) $M=4000$. The red and green dots represent the right-moving and left-moving ants, respectively.

this scaling relation numerically as in shown in Fig. 5. During a collision each loose cluster "shreds" the oppositely moving cluster; both clusters shred the other equally if $\phi=1 / 2$ (Fig. 6(a)). However, for all $\phi \neq 1 / 2$, the minority cluster suffers more severe shredding than that suffered by the majority cluster (Fig. 6(b)) because each member of a cluster contributes in the shredding of the oppositely moving cluster.

Fig. 7(a) and 7(b) show magnified view of the first and second collision area respectively in the space-time plot Fig. 6(c) where only right moving ants are present. Fig. [7(a) and 7(b) demonstrate that the phenomenon of shredding is such that the total number of clusters, their sizes as well as their individual velocities just after the collision are usually 


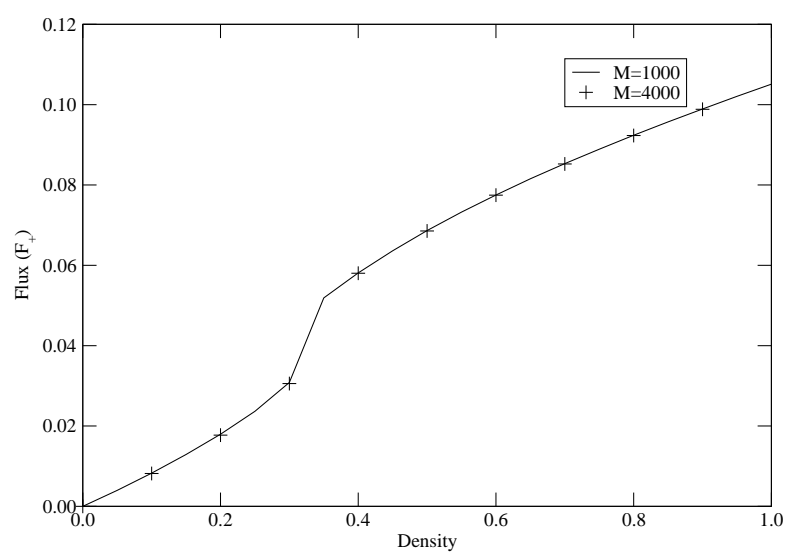

Figure 9. Fundamental diagrams for the system size $M=1000$ and $M=4000$; common parameters being $c=0.2, \phi=0.3, Q=0.75, q=0.25, K=0.50$ and $f=0.005$



Figure 10. Average size of the cluster $R$ plotted against time $t$ for $\phi=1.0$, and $\phi=0.5$, both for the same total density $c=0.2$; the other common parameters being $Q=0.75, q=0.25, K=0.50, f=0.005, M=4000$. Dotted line corresponds to a slope of $t^{1 / 3}$ and dashed line corresponds to a slope of $t^{1 / 2}$.

different from those of the clusters before the collision. But, at present, we do not have any theory to predict the changes in these properties caused by the collision.

In small systems the "shredded" clusters get opportunity for significant re-coarsening before getting shredded again in the next encounter with the oppositely moving particles. But, in sufficiently large systems, shredded appearance of the clusters persists. This is demonstrated clearly by the space-time plots for two different system sizes in Fig. 8. However, we observed practically no difference in the fundamental diagrams for $M=1000$ and $M=4000$ (see Fig. 9). Following the methods of ref. [21], we have computed $R(t)$ starting from random initial conditions. The data corresponding 
to $\phi=1$ are consistent with the asymptotic growth law $R(t) \sim t^{1 / 2}$. In the begining $R(t)$ grows as $t^{1 / 3}$ (dotted line in Fig. 10 corresponds to a slope of $t^{1 / 3}$ ) however in the later stage it grows as $t^{1 / 2}$ (dashed line in Fig. 10 corresponds to a slope of $t^{1 / 2}$ ). In sharp contrast, for $\phi=0.5, R(t)$ saturates to a much smaller value that is consistent with highly shredded appearance of the clusters in Fig. 6(a).

Thus, coarsening and shredding phenomena compete against each other and this competition determines the overall spatio-temporal pattern. Therefore, in the late stage of evolution, the system settles to a state where, because of alternate occurrence of shredding and coarsening, the typical size of the clusters varies periodically. Moreover, comparing Fig. 6(b) and Fig. 6(c), we find that, for given $c$ and $\phi$, increasing $K$ leads to sharper speeding up of the clusters during collision so long as $K$ is not much smaller than $q$. Both the phenomena of shredding and speeding during collisions of the oppositely moving loose clusters arise from the fact that, during such collisions, the dominant process is the exchange of positions, with probability $K$, of oppositely-moving ants that face each other.

\section{Conclusions}

The $P R L$ model reported in this paper, is motivated by bi-directional ant traffic. In a spatially constrained situation, e.g., on a hanging cable (Fig (1), such a single-lane model is adequate, whereas otherwise a full two-lane model 24 is required.

The main effect of the new species of particles $P$ is that coupling of its nonconserving dynamics with the conserved dynamics of the $L$ and $R$ species of particles gives rise to an effective pheromone-mediated interaction between the particles of the same species. This pheromone-mediated interactions between the $L(R)$ particles gives to a left-moving (right-moving) cluster. This tendency for "coarsening", induced by the pheromones, then competes with the "shredding" process which occurs during collision of oppositely moving clusters in a finite system with periodic boundary conditions.

The most surprising finding is a nontrivial and, at first sight, counter-intuitive, system size dependence of the spatio-temporal organization which, we believe, could be relevant also for other driven systems with competing aggregation and disintegration. It would be interesting to obtain a more quantitative description of the shredding process that allows to quantify this size dependence. Work in this direction is currently under progress.

\section{Acknowledgments}

We thank Martin Burd, Madhav Gadgil, Raghavendra Gadagkar, Alexander John and Joachim Krug for enlightening discussions. 
Competition of coarsening and shredding of clusters in a driven diffusive lattice gas 11

\section{References}

[1] B. Schmittmann and R.K.P. Zia, in: Phase Transitions and Critical Phenomena, Vol.17, eds. C. Domb and J.L. Lebowitz (Academic Press, 1995).

[2] G. Schütz, in: Phase Transitions and Critical Phenomena, Vol.19, eds. C. Domb and J.L. Lebowitz (Academic Press, 2000).

[3] D. Mukamel, in: Soft and Fragile Matter: Nonequilibrium Dynamics, Metastability and Flow, p. 205, eds. M.E. Cates and M.R. Evans (IOP Publishing, Bristol 2000).

[4] M.R. Evans, D.P. Foster, C. Godreche and D. Mukamel, Phys. Rev. Lett. 74, 208 (1995); J. Stat. Phys. 80, 69 (1995).

[5] M.R. Evans, Y. Kafri, H.M. Koduvely and D. Mukamel, Phys. Rev. Lett. 80, 425 (1998); Phys. Rev. E 58, 2764 (1998); M. Clincy, B. Derrida and M.R. Evans, Phys. Rev. E 67, 066115 (2003).

[6] P. F. Arndt, T. Heinzel and V. Rittenberg, J. Phys. A 31, L45 (1998); J. Stat. Phys. 97, 1 (1999).

[7] N. Rajewsky, T. Sasamoto and E.R. Speer, Physica A 279, 123 (2000).

[8] R. Lahiri, M. Barma and S. Ramaswamy, Phys. Rev. E 61, 1648 (2000).

[9] L. Frachebourg, P.L. Krapivsky and E. Ben-Naim, Phys. Rev. E 54, 6186 (1996).

[10] V. Popkov and G.M. Schütz, J. Stat. Phys. 112, 523 (2003)

[11] Number of species would be four if vacancies are also regarded as a different species.

[12] B. Hölldobler and E.O. Wilson, The ants (Belknap, Cambridge, USA, 1990).

[13] D. Chowdhury, L. Santen and A. Schadschneider, Phys. Rep. 329, 199 (2000).

[14] D. Helbing, Rev. Mod. Phys. 73, 1067 (2001)

[15] M. Burd, D. Archer, N. Aranwela and D. J. Stradling, American Natur. 159 (2002) p.283.

[16] M. Burd and N. Aranwela, Insect. Sociaux 50, 3 (2003)

[17] I.D. Couzin and N.R. Franks, Proc. Roy Soc. London B 270, 139 (2003).

[18] D. Chowdhury, V. Guttal, K. Nishinari and A. Schadschneider, J. Phys. A:Math. Gen. 35, L573 (2002).

[19] K. Nishinari, D. Chowdhury and A. Schadschneider, Phys. Rev. E 67, 036120 (2003).

[20] O.J. O'Loan, M.R. Evans and M.E. Cates, Phys. Rev. E 58, 1404 (1998).

[21] D. Chowdhury and R.C. Desai, Eur. Phys. J. B 15, 375 (2000).

[22] D. Helbing, F. Schweitzer, J. Keltsch and P. Molnar, Phys. Rev. E 56, 2527 (1997); see also [14].

[23] C. Burstedde, K. Klauck, A. Schadschneider and J. Zittartz, Physica A 295, 507 (2001).

[24] A. John, A. Schadschneider, D. Chowdhury and K. Nishinari, J. Theor. Biol. 231, 279 (2004). 